B. BARRED SPIRALS 


\title{
68. BARRED SPIRAL GALAXIES
}

\author{
K. C. FREEMAN \\ Australian National University, Canberra, Australia
}

\begin{abstract}
We point out some properties of barred spiral galaxies which are important for the theory of their formation and spiral structure, and describe some theoretical work on the dynamics of these systems.
\end{abstract}

\section{Some Observed Properties}

What are barred spirals? They are members of the family of disk-like galaxies which includes all spirals and lenticulars (SO), and they have an inner bar structure; many examples are shown in The Hubble Atlas of Galaxies (Sandage, 1961). Apart from this bar and associated features, such as the dustlanes, they are similar in structure and content to non-barred systems.

Two remarks: (a) There is no clear division between barred and non-barred systems; the transition SA-SAB-SB at any stage is smooth (de Vaucouleurs, 1959). (b) Barred spirals are not rare oddities of nature. Among 994 bright spirals, about $\frac{2}{3}$ show bar structure $(31 \% \mathrm{SA}, 28 \% \mathrm{SAB}, 37 \% \mathrm{SB}, 4 \% \mathrm{~S}$; see de Vaucouleurs, 1963a). If one plans to discuss the dynamics of disk galaxies, then it must be clear that axisymmetry is the exception, not the rule.

On most reproductions, the bar looks dominant in size and luminosity, but this impression is not usually correct. Surface photometry shows very clearly that the bar is a relatively small feature immersed in an approximately axisymmetric disk. Table I gives the NGC number, the morphological type, the ratio $L_{\mathrm{B}} / L_{\mathrm{T}}$ of the bar luminosity to the total luminosity, the ratio $a_{\mathrm{B}} / a_{27.0}$ of the bar length to the disk diameter at a surface brightness of 27.0 magnitudes $\operatorname{arc~sec}^{-2}$, and the source of the photometry, for three barred galaxies.

\section{TABLE I}

Data for three barred spirals

\begin{tabular}{lllll}
\hline NGC & Type & $L_{\mathrm{B}} / L_{\mathrm{T}}$ & $a_{\mathrm{B}} / a_{27.0}$ & Source \\
\hline & & & & \\
1313 & SB(s)d & 0.12 & 0.15 & de Vaucouleurs (1963b) \\
6744 & SAB(r)bc & 0.10 & 0.10 & de Vaucouleurs (1963c) \\
1433 & SB(r)a & 0.15 & 0.30 & de Vaucouleurs (unpublished)
\end{tabular}

I emphasise that, in the luminosity distribution, the asymmetry associated with the bar is limited to the inner part of the system; most of the light comes from the approximately axisymmetric disk. First I discuss this disk, and then the bar.

The disks of SB systems have in general an exponential radial luminosity distribution $I(R)=I_{0} \exp (-\alpha R)$, and differ in no way from the disks of SA systems. For example, the parameters $I_{0}$ and $\alpha$ change with classification stage $(a, b, \ldots, m)$ in the 
same way for the disks of SA and SB galaxies (see Freeman, 1970). As in SA systems, the spiral arms in blue light are defined mainly by $\mathrm{H}_{\mathrm{II}}$ regions, hot stars, and dust. Little is known about the involvement of the old disk population (which usually contains most of the mass) in the spiral structure. The author's infrared plates of M83, and Garrison's visual plates of many spirals (see Morgan, this volume, p. 9) show relatively amorphous spiral arms underlying the highly resolved blue light structure. Are these 'red arms' just a slightly older population I feature, or do they involve the old disk itself? In Baade's picture, is the spiral structure just frosting on the cake, or do we have a spiral cake? It seems important for understanding spiral structure that this point be cleared up: unfortunately it is not obvious that it can be done observationally, even in principle.

What is the nature of the bar, i.e. to what population does it belong? In some earlier type systems (e.g. NGC 6744), the bar follows the luminosity distribution $\log I(R)$ $\propto R^{1 / 4}$, which appears to be characteristic of the spheroidal component in SA galaxies (de Vaucouleurs, 1959). There is evidence that the bar is imbedded in the spheroidal component for systems earlier than about $\mathrm{SBc}$; the bar colours and spectra are consistent with this picture. For later type galaxies, the bar nature is not clear; in NGC 55, a nearly edge-on SBm system (de Vaucouleurs, 1961), the bar is no more extended in the direction perpendicular to the galactic plane than is the disk. At least for the earlier type systems, we identify the bar photometrically and spectroscopically with the old spheroidal population.

Two remarks about the bar: (a) Note the characteristic dust lanes along the bar in earlier type SB systems like NGC 1300. These probably have some dynamical importance which is not yet understood. (b) Systems earlier than about SBc have the bar central in the disk. At about SBd a second asymmetry appears: the bar becomes displaced from the disk centre. This effect is discussed by de Vaucouleurs and Freeman (this volume, p. 356).

Finally I discuss the velocity field in barred galaxies. Apart from rotation, the $\mathrm{HII}$ velocity field shows noncircular motions in many SB systems, at least in the nuclear regions. In some late type barred spirals, there appears to be a gas flow outwards along the bar, with velocities of order $100 \mathrm{~km} \mathrm{~s}^{-1}$ and mass flux of order $1 M_{\odot} \mathrm{yr}^{-1}$. NGC 4027 (de Vaucouleurs et al., 1968) is a particularly good example, because its bar is almost exactly perpendicular to its line of nodes; it is then easy to distinguish streaming motions along the bar from the general rotation field.

To summarise this section: The main difference between the A and B families of spirals is the departure from axisymmetry in the inner parts of B galaxies. It seems that the basic problems for the two families are similar: the formation and dynamics of the old background disk + spheroidal (+bar) components, and the formation and maintenance of the spiral structure.

\section{Theoretical Work}

I describe a gravitational theory which appears to have some success in explaining 
features of SB galaxies, although its relevance is limited to these systems. I do not consider the disk here, because its problems are common to all disk galaxies. First I discuss the bar.

The formation of the bar may not be a fundamental problem. Several authors have shown how the collapse of uniform density spheroids (rotating or not) tends to produce prolate figures if the ratio (gravitational energy)/(internal energy) is fairly large. If it is true that the bar is part of the old spheroidal component, then this ratio may determine whether the spheroidal component stays axisymmetric, as in SA galaxies, or develops a bar structure.

Little is known about the stellar dynamics of realistic steady state bar-like systems. However the study of unrealistic models seems to be very instructive. Consider a general uniformly rotating bar, in a steady state referred to the rotating frame. It follows from the collisionless Boltzmann equation that the distribution function $f$ depends only on the only isolating (Jacobi) integral $J=\frac{1}{2} c^{2}-\Phi_{1}$, where $\Phi_{1}$ is the potential for the centrifugal + gravitational force field. The average velocity $\overline{\mathbf{c}}$ of stars, referred to the rotating frame, is then identically zero because

$$
\int c f(J) \mathrm{d}^{3} c \equiv 0
$$

by the symmetry of $J$ in $\mathbf{c}$.

Now consider a special bar model. It is possible to construct uniform density ellipsoidal stellar systems, rotating rigidly about a principal axis (Freeman, 1966). These are completely self-consistent exact solutions of the collisionless Boltzmann equation and Poisson's equation. (It is not known whether they are stable.) Because of the properties of uniform ellipsoids, $J$ is not the only isolating integral, so $\overline{\mathbf{c}}$ is not identically zero. It turns out that the mean stellar motion shows a strong circulation counter to the sense of rotation, just as in the fluid Dedekind ellipsoids. The circulation is so strong that near the minor axes of the bar, the tangential velocity of the stars in the bar is in the opposite sense to the rotation of the figure of the bar. In NGC 4027, which is ideally orientated (minor axis of the bar along the line of nodes), this remarkable predicted effect has been observed (de Vaucouleurs et al., 1968). The effect was evident in 7 stellar absorption lines in 3 independent spectograms, so it is probably real. The relevance of this effect to the problem of the Symposium is that, for constructing zero-order background models of barred systems, it is probably incorrect to take $f=f(J)$ alone.

I now discuss the spiral arms and ring structures (e.g. NGC 1433, de Vaucouleurs, 1959) of SB systems. Most of the theoretical work peculiar to barred galaxies depends on the properties of test particle orbits in the gravitational field of a rotating bar.

(i) The spiral arms. Several authors have described spiral structure formation based on the gas outflow observed along the bars of some SB systems. There are some interesting results: (a) Model the bar by a rapidly rotating ellipsoid of typical mass and dimensions. The gravitational torque of this bar can support an outflow along the bar, against the Coriolis force, of order $100 \mathrm{~km} \mathrm{~s}^{-1}$, as observed. (b) Fol- 
low numerically these outflowing particles after they leave the bar: they define quite realistic spiral arms as long as the outflow continues (see e.g. Freeman, 1965).

Julian and Toomre (1966) suggest that a bar rotating in a differentially rotating disk of stars may set up a forced trailing density wake response in the disk from the bar ends. This suggestion raises again the question of the involvement of the old disk in the spiral structure.

(ii) The ring structure, observed in about $25 \%$ of SB systems. It seems possible to account for this structure from the properties of particle orbits in the gravitational field of a rotating bar. Consider a prolate spheroidal bar alone, axial ratio $\simeq 5$, mass $\mathscr{M}$, semi-length $L$, and uniform angular velocity $\Omega$. There is one dimensionless number, $Q=3 G \mathscr{H} /\left(\Omega^{2} L^{3}\right)$. (The breakup angular velocity corresponds to $Q \simeq 1$.) For a fairly slowly rotating bar $(Q \simeq 15)$, there exists a family of approximately circular periodic orbits, encircling the bar near bar ends. These orbits lead the bar slightly, are highly stable, and cover a fairly wide annulus (width $\simeq L / 4$ ) around the bar (see de Vaucouleurs and Freeman, 1970, for full details). It can be shown numerically, from surface of section studies, that it is easy to trap enough matter near these stable periodic orbits to produce the apparent increase in luminosity associated with the observed rings. However, as $Q$ is decreased, the annulus covered by the stable circular periodic orbits becomes less wide, and the matter trapping efficacy decreases. For $Q \lesssim 8$, the periodic orbits no longer exist, and the trapping of matter to form the ring structure is no longer possible. In this picture, the $\mathrm{SB}(\mathrm{r})$ systems are slower rotators, in a dimensionless sense, than the $\mathrm{SB}(\mathrm{s})$ systems. There is no real observational evidence yet either for or against this inference.

The particle orbit theory for the spiral structure and rings in SB galaxies, and for some of the features of the highly asymmetric SBm systems (see de Vaucouleurs and Freeman, this volume, p. 356) seems to have some success, and probably points to some relevant dynamical process. However I am uneasy about any theory for galactic structures that does not work for all galaxies, SA or SB; the transition SA-SAB-SB is so smooth that it seems inconceivable that the structure producing processes should be essentially different for SA and SB systems. I do not believe that we can claim to understand these processes until we find one that works for all systems, barred and unbarred.

\section{References}

Freeman, K. C.: 1965, Monthly Notices Roy. Astron. Soc. 130, 183.

Freeman, K. C.: 1966, Monthly Notices Roy. Astron. Soc. 134, 1.

Freeman, K. C.: 1970, Astrophys. J., in press.

Julian, W. and Toomre, A.: 1966, Astrophys. J. 146, 810.

Sandage, A. R.: 1961, The Hubble Atlas of Galaxies, Carnegie Institution of Washington Washington.

Vaucouleurs, G. de: 1959, Handbuch der Physik 53, 275.

Vaucouleurs, G. de: 1961, Astrophys. J. 133, 405.

Vaucouleurs, G. de: 1963a, Astrophys. J. Suppl. Ser. 8, No. 74, 31.

Vaucouleurs, G. de: 1963b, Astrophys. J. 137, 720.

Vaucouleurs, G. de: 1963c, Astrophys. J. 138, 934. 
Vaucouleurs, G. de, and Freeman, K. C.: 1970, 'Structure and Dynamics of Barred Spiral Galaxies, and in particular of the Magellanic Type', in Vistas in Astronomy (ed. by A. Beer), to be published.

Vaucouleurs, G. de, Vaucouleurs, A. de and Freeman, K. C.: 1968, Monthly Notices Roy. Astron . Soc. 139, 425 .

\section{Discussion}

Miller: Your arguments lean heavily on photometry at light levels well below that of the night sky, and seem to imply much more accurate subtractions of the night sky than can usually be justified.

Freeman: Comparison of detailed isophotometry of several objects by independent observers using independent plate material and calibrations indicates that acceptably accurate photographic surface photometry to at least three magnitudes below the night sky level can be achieved.

Toomre: In his very nice lecture on barred spirals Ken Freeman also touched on a vital clue to the ordinary spirals. As he said, and as De Vaucouleurs especially has emphasized, it is remarkable that there exists no sharp break between the barred and the obviously disk-like galaxies. Instead, their transition appears to be almost continuous, and upon closer inspection, not only do more and more of the bars, like that of the LMC, turn out to be imbedded in fairly disk-like systems, but even some galaxies as 'normal' as M31 seem to exhibit internal motions suggesting some sort of a bar-like asymmetry.

As a theoretician, I am delighted with this trend of evidence for two reasons: For one thing, I am now convinced that Lin's 'asymptotic' theory of spiral density waves suffers from the serious - but probably non-fatal - difficulty that such already tightly wound waves need continually to be replenished, apparently from length scales so large that the present form of that theory ceases to be applicable. (Because of a group velocity inherent to such waves, I might add, this need for replenishment is rather more acute than Oort surmised in his opening lecture.) On the other hand, it also seems clear from work beginning with the sheared density waves of Goldreich and Lynden-Bell, that every differentially rotating galactic disk is very responsive to excitation. Now this forcing could come tidally from a nearby galaxy, as I discussed briefly in my own talk. Or it could arise from large gas complexes orbiting within the disk itself, as Julian and I once demonstrated. But an even more impressive source of these forced spiral waves should be the gravity forces from any slowly rotating bar-like or oval distortion involving a major (and probably central) fraction of the mass of a galaxy.

I do not know, of course, that any given disk is so obligingly distorted. But Freeman's remarks, Lin's dilemma, this spiral responsiveness, and numerical calculations such as those of Kalnajs all suggest that at least a mild case of the bar disease afflicts most of the seemingly ordinary spiral galaxies. 